\title{
Anthropic interpretation of quantum theory
}

\author{
Brandon Carter \\ LuTh, Observatoire de Paris, 92195 Meudon
}

July, 2003

\begin{abstract}
The problem of interpreting quantum theory on a large (e.g. cosmological) scale has been commonly conceived as a search for objective reality in a framework that is fundamentally probabilistic. The Everett programme attempts to evade the issue by the reintroduction of determinism at the global level of a "state vector of the universe". The present approach is based on the recognition that, like determinism, objective reality is an unrealistic objective. It is shown how an objective theory of an essentially subjective reality can be set up using an appropriately weighted probability measure on the relevant set of Hilbert subspaces. It is suggested that an entropy principle (superseding the weak anthropic principle) should be used to provide the weighting that is needed.
\end{abstract}

Text for exposition presented at Interdisciplinary Colloquium "La Philosophie de la Nature aujourd'hui?", Paris, March 2003, and 8th Peyresq Physics Meeting "The Early Universe", June 2003. 


\section{Introduction}

Among the diverse schools of thought concerning the interpretation of quantum theory, it is the one founded by Everett [1] that can claim the widest nominal adherence [2] (if not an absolute majority, surely more than for any specific alternative) within the theoretical physics community. Those who are not satisfied by the Everett doctrine belong to two distinct groups. The first (including perhaps a majority of experimental, as opposed to theoretical, physicists) consists of those who have not thought about the issue, and who are content with earlier Copenhagen type interpretations such as are useful in a restricted laboratory context but inapplicable in a broader, e.g. cosmological, context. The others, namely those who are not so content (and who have often been inclined to search - vainly - for acceptable alternatives of a deterministic nature) are of two kinds, of which perhaps the most numerous consists of those who dislike the prolificity implicit in Everett's "many world" idea. The remaining minority consists of those who have looked into the matter sufficiently carefully to be aware of the intrinsic intellectual deficiency of the Everett doctrine.

In order to satisfy the requirements of the latter group, namely those who are broad minded about ontology, but more rigourously demanding in so far as intellectual coherence is concerned, the alternative interpretation proposed here is based on a rather different approach that incorporates ideas developed particularly by Dyson [3] and by Page [4.

\section{Deficiency of the Everett interpretation}

It has been recognised on many occasions that Everett's doctrine is intrinsically self contradictory if taken too literally, while on the other hand, if taken less literally, the Everett "interpretation" is so ambiguous as to be essentially meaningless unless it is itself provided with an appropriately coherent interpretation, of which various kinds [5] have been envisaged

As in more traditional approaches, the starting point of the Everett doctrine is the generally accepted principle that observational discrimination is describable in quantum theory as a choice between mutually orthogonal Hilbert subspaces, which are describable as the alternative branch-channels, each of which is characterised by a corresponding Hilbert space projection operator $\mathbf{e}=\mathbf{e}^{2}$.

It is to be noted that many writings, including those of Everett, convey the potentially misleading idea that the number of such "branches" will automatically multiply as successive observations are made. To see that, as emphasised by Tippler [6], this need not - and indeed, beyond a certain point, ultimately cannot - be the case, it suffices to consider the usual kind of toy sensor model, in which what is observed is a combination of up or down spin orientations (with respect, for example, to a background magnetic field direction) for a set of let us say $S$ sensor particles. For a single particle initially in a pure - let us say down - state, an elementary measurement will indeed double the number of relevant branch channels, but such doubling can give rise to at most a maximum value $\mathcal{N}=2^{S}$ of branch-channels, corresponding to the recording of a maximum of $S$ bits of information. Subsequent observations can record new information only at the expense of erasure (by conversion [7] into Landauer entropy) of part of what was previously recorded - unless of course the number $S$ is itself increased. Extrapolating from the example of such a toy to the case of a human observer, it is to be remarked that the relevant information capacity $S$ will indeed increase progressively during infancy (though not quite fast enough to prevent children from forgetting as well as learning) but that it will saturate 
during adulthood (and ultimately decrease if a stage of senility is attained).

Whether their number is increasing or not, a set of probabilities for such alternative branch-channels is supposed, in the traditional approach, to be provided by a prescription whereby any particular branch channel, $e$ say, will have probability

$$
P_{[\mathcal{O}]}\{e\}=\operatorname{tr}\{\mathbf{P} \mathbf{e}\},
$$

given by the appropriate choice of a von Neumann type (unit trace) operator, $\mathbf{P}$, which (as in the classical Bayesian case) is readjusted to take account of new observational information as and when it becomes available. It is to be noted that this is just a conditional probability, subject to the requirement that the relevant observation, $\mathcal{O}_{e}$ say, be actually carried out. Further information must be supplied (as discussed below) if one needs an absolute probability $P\{e\}$ allowing for the unlikelihood of that particular observation ever really being performed.

This works very well in a laboratory context for an observer outside the system under consideration, but in more general (e.g. cosmological) contexts, involving more than one observer inside the system, the desideratum of using a probability distribution that treats distinct observers (having access to different information) on the same objective footing poses a problem.

The Everett strategy for dealing with the problem of the subjectivity of observational readjustment of the probability distribution is to deny any objective significance for observational discrimination by proclaiming [1] that all the relevant branch-channels are "equally real". The trouble with this is with this is that, if taken at its face value, it disagrees with ordinary laboratory experience, for which the probability interpretation works so well. In what Graham describes [8] as an attempt to to "escape from this dilemma" Everett is obliged to resort to an Orwellian gambit whereby the meaning of of "reality", and of "equality", is cast into doubt by the admission that the branch-channels thus described are nevertheless characterised by different "weightings". Although they are supposed to be given in the usual way by a unit trace operator $\mathbf{P}$, these "weightings" are deemed not to be ordinary subjective probabilities but to have an objective physical status inherited from a physically "real" state vector $|\Psi\rangle$ of the universe, in terms of which the "weighting" operator has the pure state form $\mathbf{P}=|\Psi\rangle\langle\Psi|$.

Opinions may differ about the most appropriate choice of the cosmological state function [10, 11] and about whether the ontological prolificity entailed by the "many worlds" idea is appealing or appaling [9], but what is intrinsicly wrong with Everett's so called "interpretation" is his failure to provide a self-consistent interpretation of the terms such as "weighting" and particularly "reality" - on which his formulation relies.

The approach described below provides a remedy in which the concept of "reality" is interpreted in a more realistically restrictive manner, while the "weightings" are reinstated in their traditional role as (conditional) probabilities in the usual (Born) sense.

\section{A viable approach}

Although other kinds of reality can be imagined (e.g. by theologians) the only reality of which we have a direct knowledge is that of a subjective mental perception, which can of course include memories of previous perceptions, which - as discussed by Page [4] - are collectively construed as mind states of the perceptor. Many such mind states are interpretable merely as dreams, but many more are interpretable as corresponding to events 
in an extrinsic physical world. The aim of the natural (physical and biological) sciences is to understand how this physical world works and how our perceptions fit into it. These sciences provide descriptions of various aspects of the extrinsic world in terms of more or less elaborate theoretical models in which events that are actually supposed to occur are selected within a wider class of eventualities that may or may not occur. (For example a kind of toy model that is particularly popular for pedagogical and other purposes in theoretical physics consists of a scalar field governed by a hyperbolic differential equation whose solutions are the eventualities, of which a particular member is selected as an actual event by some specific choice of initial conditions.)

It is sometimes useful to employ a model of the simplified kind (exemplified by that of the traditional criminal jury, for which the only admissible eventualities are "guilty" and "not guilty") that is qualifiable as deterministic, meaning that the specification of the events that actually occur is provided in an unambiguous manner. However it is often more realistic to employ a model that is indeterminate, in the sense that it leaves the question of which eventualities occur as actual events to be decided (or not, as the case may be) by a process of observation and perception that may (subsequently) take place outside the framework of the model under consideration. In the most effective kind of indeterminate model, a guide to what is most likely to be (subsequently) observed is provided by the attribution to the relevant eventualities of a probability weighting that is conceived (there is a vast literature on this subject) as a generalisation of the relative frequency that would be obtained if (as is seldom the case) it were possible perform an unlimited number of observation and perception processes under identical conditions.

Among such probabilistic models, a particularly important category is that of quantum theoretical models, which provide the most fundamental kind of physical description available today. This means that other deterministic or probabilistic descriptions (such as were sufficient for describing what was known before the end of the nineteenth century) can be considered just as approximations to an underlying quantum description (which at some future epoch may itself turn out to be qualifiable as an approximation to something even more mysterious that has not yet been conceived, and for which there is as yet no obvious necessity). In a quantum theory, an admissible eventuality, $e$ say, is supposed to be identifiable with a corresponding Hilbert subspace, for which a conditional probability weighting is provided by a von Neumann type operator, $\mathbf{P}$ say, according to a prescription of the form (11).

Since the various conceivable eventalities will in general not be mutually exclusive, the sum of their conditional probabilities $P_{[\mathcal{O}]}\{e\}$ can greatly exceed unity. A well behaved probability measure $P\{e\}$ on the set of Hilbert subspaces will however be obtainable if a probability $P\left\{\mathcal{O}_{e}\right\}$ for the actual occurrence of a corresponding observational discrimination (between $e$ and a set of mutually orthognal alternatives that together form an "observable" set completely spanning the Hilbert space of the system) is provided within a suitably extended framework that includes an appropriate sensor system. The probability $P\{e\}$ for the eventuality $e$ to be actually observed will then be given by

$$
P\{e\}=P\left\{\mathcal{O}_{e}\right\} P_{[\mathcal{O}]}\{e\} .
$$

Of course for most of the so called "observables" in physical models, such an actual observation would be practically impossible or at best highly improbable, so that the relevant factor $P\left\{\mathcal{O}_{e}\right\}$ would be negligibly small. 
In order to use an abstract physical model as a guide to "reality", meaning [4 what is actually perceived, it is of course necessary to postulate that there should be a correspondence between the mind of the perceptor and the brain of a physical observer. It is to be understood that the latter is identifiable within the model as a physical sensor subsystem, for which there is a special subclass of eventualities - called perceptibles - of the particular kind to which the the only events that are undisputably real - namely those identifiable with actual perceptions - must belong.

In order to avoid attribution of a privileged status to some particular physical observer such as Schroedinger (the performer of the famous cat experiment) or (like many a subconscious solipsist) oneself, it is necessary to situate the probabilistic (quantum or even classical) physical model within the framework of an extended perceptual system in which the perceptor has the role of an ultimate sensor of sensors. The eventualities within this perceptual system consist, not just of those within the underlying quantum mechanical system, but in addition, for each perceptible eventuality $e$ there is a corresponding perceptual eventuality, $\mathcal{O}_{e}$ say, namely that of being being subject to an actual observational discrimination by the perceptor. To complete the structure of the perceptual theory, all that remains is to specify a corresponding distribution of probabilities $P\left\{\mathcal{O}_{e}\right\}$ (that must vanish for non-perceptible eventualities) in a manner that avoids giving a priori privilege to one's own situation.

When this is done, the probability, $P\{e\}$ say, for a particular perception to be the one corresponding to the physical eventuality $e$ will be given by an expression of the form (2) in which $P_{[\mathcal{O}]}\{e\}$ is the ordinary physical probability as given, according to (11) by the underlying quantum mechanical model, while the factor $P\left\{\mathcal{O}_{e}\right\}$ is something that must be provided by an ansatz that appropriately refines the original (weak) anthropic principle [12, 13] whereby equal weight was attributed to comparable anthropic observers.

\section{Entropic principle.}

For the present purpose it is necessary not just to attribute a probability weighting to the entire life of an observer qualifiable as anthropic (in the sense of being sufficiently similar to ourselves) but to individual perceptions in the lives of observers who may be very different. It is to be reiterated that these perceptions are not just simple events (like the recognition that one's cat is or is not still alive) but that they may involve an intricate web of memories corresponding to something at least as complicated as what are known as consistent histories [14].

A first idea that comes to mind is to take the required weighting $P\left\{\mathcal{O}_{e}\right\}$ for such a perception to be proportional to its proper time duration, which in our human case is generally considered [4] to be of the order of a fraction of a second. However the line of reasonning developped in Dyson's discussion [3] of other conceivable life forms suggests that a more appropriate measure is that of the amount of information, $S_{e}$, effectively processed during the perception.

The relevant information value, $S_{e}$ say, may be roughly estimated as the information capacity of the relevant number $\mathcal{N}_{e}$ of Everett type branch-channels (meaning the maximum of the Shannon entropy obtained by summing $-P \log \{P\}$ for a probability distribution $P$ over the branch-channels) which will be given simply by

$$
S_{e}=\log \left\{\mathcal{N}_{e}\right\}
$$

(using a logarithm with base 2 for ordinary bit units). If it is supposed that the relevant 
branch-channels all have the same dimension, which will be given by the rank of the projection operator e associated with the perception, namely its $\operatorname{trace} \operatorname{tr}\{\mathbf{e}\}$, then in a Hilbert space with dimension $\operatorname{tr}\{\mathbf{I}\}$ (where $\mathbf{I}$ is the identity operator) the required branchchannel number will be given by

$$
\mathcal{N}_{e}=\operatorname{tr}\{\mathbf{I}\} / \operatorname{tr}\{\mathbf{e}\}
$$

(It is to be noted that this ratio $\mathcal{N}_{e}$ is unaffected by the scaling up process that will occur if the Hilbert space for a local system is extended to the higher dimensional Hilbert space needed for describing a larger chunk of the universe. It is also to be noted that it is multiplicative, so that the logarithm (3) will be additive, when perceptions by distinct individuals are merged to form the perception of a team or of an entire civilisation. )

Dyson estimated that the total entropy $Q_{e}$ generated during a human perception would be of the order of Avogadro's number, but that includes metabolic processes throughout the body, and so exceeds the Landauer entropy resulting [7] from the relevant mental information $S_{e}$ by an enormous thermodynamic waste factor, $W_{e}=Q_{e} / S_{e} \gg 1$.

The ansatz proposed here, namely

$$
P\left\{\mathcal{O}_{e}\right\} \propto S_{e}
$$

with

$$
S_{e}=\log \{\operatorname{tr}\{\mathbf{I}\}\}-\log \{\operatorname{tr}\{\mathbf{e}\}\},
$$

may appropriately be designated by the term entropic principle (paraphrasing the cruder anthropic principle that it supersedes). It is to be hoped that future investigations (of a neurological nature) may provide estimates of the absolute value of the relevant mental information $S_{e}$ that are more precise than the vague indication provided here (namely that in the adult human case it is very large compared with unity and very small compared with Avogadro's number) and that may be able to evaluate the decrease that presumably occurs during a transition from a waking to a dreaming state. However the importance of such uncertainties (and of the even greater uncertainty concerning the proportionality factor in (5), which is affected by the weighting that may be attributed to conceivable extraterrestrials) is diminished by the consideration that many applications require only relative probabilities, not absolute probability values.

Substitution of (11) and (5) in (2) leads to a prescription giving the probability of a perceptible eventuality $e$ in the final form

$$
P\{e\} \propto S_{e} \operatorname{tr}\{\mathbf{P} \mathbf{e}\},
$$

with $S_{e}$ given by (6). The kind of approach encapsulated in this formula allows considerable latitude for adaptation to satisfy idealogical desiderata of various alternative kinds, such as belief that the von-Neumann operator $\mathbf{P}$ should be obtained from a pure state function $|\Psi\rangle$ of the universe [10, 11], and prejudice for or against [9] ontological prolificity. For example as formulated above, in terms of a single (though far from simple 4]) act of perception, the interpretation presented here may not entirely satisfy those who demand that probabilities be defined in terms of frequencies. However that can easily be remedied by supposing that a very large number of perceptions is made (by one or many perceptors). So long as it does not affect the values of the distributions given by 
$P\left\{\mathcal{O}_{e}\right\}$ and $\mathbf{P}$, such a multi-perceptual reinterpretation makes no difference for practical (purely scientific) predictive purposes, though it might be slightly less satisfactory with respect to Ockham's "razor" criterion and much less satisfactory from the point of view of ontological economy.

The author wishes to thank Martin Rees for hosting the meeting at which this proposal was first informally mooted.

\section{References}

[1] H. Everett, "Relative state formulations of quantum theory", Rev. Mod. Phys. 29 (1957) 454-462.

[2] M. Tegmark, J.A. Wheeler, "100 years of the quantum", Sci. Am. 284 (2001) 68-75. quant-ph/0101077

[3] F.J. Dyson, "Time without end: physics and biology in an open system", Rev. Mod. Phys. 51 (1979) 447-460.

[4] D. Page, "Sensible quantum mechanics: are probabilities only in the mind?", Int. J. Mod. Phys. D5 (1996) 583-596. gr-qc/9507024

[5] J.A. Barrett, "On Everett's interpretation of Quantum Mechanics", The Monist 80 (1997) 70-76.

[6] "The many worlds interpretation of quantum mechanics in quantum cosmology", in Quantum concepts in space and cosmology, ed. R. Penrose, C.J. Isham (Oxford U.P., 1986).

[7] V. Vedral, Proc. R. Soc. Lond. A456 (2000) 969-984.

[8] N. Graham, in The many worlds interpretation of quantum mechanics, ed. B.S. De Witt, N. Graham (Princeton U.P., 1973) 229-253.

[9] J. Leslie, in Scientific Understanding, ed. N. Rescher (University Press of America, Lanham and London, 1983).

[10] J.B. Hartle, S.W. Hawking, Phys. Rev. D28 (1983) 2960-2975.

[11] A. Vilenkin, Proc. Hawking Workshop, Cambridge, 2002 gr-qc/0204061

[12] B. Carter, Phil. Trans. R. Soc. Lond. A310 (1983) 347-363.

[13] N. Bostrom, Anthropic Biass: Observation selection effects in Science and Philosophy (Routlege, New York, 2002).

[14] M. Gell-Man, J.B. Hartle, in Complexity, Entropy, and the Physics of Information, ed. W.H. Zurek (Addison Wesley, Redwood City, 1991) 425-458. 